\title{
PRICING OCCUPATION-TIME OPTIONS IN A MIXED-EXPONENTIAL JUMP-DIFFUSION MODEL
}

\author{
DJILALI AIT AOUDIA AND JEAN-FRANÇOIS RENAUD
}

\begin{abstract}
In this short paper, in order to price occupation-time options, such as (doublebarrier) step options and quantile options, we derive various joint distributions of a mixedexponential jump-diffusion process and its occupation times of intervals.
\end{abstract}

\section{INTRODUCTION}

Let the price of an (underlying) asset $S=\left\{S_{t}, t \geq 0\right\}$ be of the form:

$$
S_{t}=S_{0} \mathrm{e}^{X_{t}}
$$

where $X=\left\{X_{t}, t \geq 0\right\}$ is a process to be specified (log-return process). For example, in the Black-Scholes-Merton (BSM) model, $X$ is a Brownian motion with drift. The time spent by $S$ in an interval $I$, or equivalently the time spent by $X$ in an interval $I^{\prime}$, from time 0 to time $T$, is given by

$$
A_{T}^{I}:=\int_{0}^{T} \mathbf{1}_{\left\{S_{t} \in I\right\}} \mathrm{d} t=\int_{0}^{T} \mathbf{1}_{\left\{X_{t} \in I^{\prime}\right\}} \mathrm{d} t
$$

Options linked to occupation times are often seen as generalized barrier options. Instead of being activated (or canceled) when the underlying asset price crosses a barrier, which is a problem from a risk management point of view, the payoff of occupation-time options will depend on the time spent above/below this barrier: the change of value occurs more gradually. There are several different options: (barrier) step options, corridor derivatives, cumulative(-boost) options, quantile options, (cumulative) Parisian options, etc. For a review, see e.g. [16].

Introduced by Linetsky [13], a (down-and-out call) step option admits the following payoff:

$$
\mathrm{e}^{-\rho A_{T}^{L,-}}\left(S_{T}-K\right)_{+}=\mathrm{e}^{-\rho A_{T}^{L,-}}\left(S_{0} \mathrm{e}^{X_{T}}-K\right)_{+}
$$

where

$$
A_{T}^{L,-}=\int_{0}^{T} \mathbf{1}_{\left\{S_{t} \leq L\right\}} \mathrm{d} t
$$

and where $\rho>0$ is called the knock-out rate. Indeed, it is interesting to note that we have the following relationship:

$$
\mathbf{1}_{\left\{\tau_{L}^{-}>T\right\}}\left(S_{T}-K\right)_{+} \leq \mathrm{e}^{-\rho A_{T}^{L,-}}\left(S_{T}-K\right)_{+} \leq\left(S_{T}-K\right)_{+} \cdot
$$

Date: March 25, 2022.

Key words and phrases. Path-dependent options, occupation times, jump-diffusion, mixed-exponential distribution. 
where $\tau_{L}^{-}=\inf \left\{t \geq 0: S_{t} \leq L\right\}$. Later, Davydov and Linetsky [6] studied double-barrier step call options, which are a generalization of double-barrier options (see [8]):

$$
\mathrm{e}^{-\rho^{-} A_{T}^{L,-}-\rho^{+} A_{T}^{U,+}}\left(S_{T}-K\right)_{+}=\mathrm{e}^{-\rho^{-} A_{T}^{L,-}-\rho^{+} A_{T}^{U,+}}\left(S_{0} \mathrm{e}^{X_{T}}-K\right)_{+},
$$

where

$$
A_{T}^{U,+}=\int_{0}^{T} \mathbf{1}_{\left\{S_{t} \geq U\right\}} \mathrm{d} t
$$

and where $\rho^{-}$and $\rho^{+}$are the knock-out rates. Expressions for the price of double-barrier step options are available in the BSM model and for single-barrier step options in Kou's model for example.

Studied by Fusai [7] in the BSM model (see also the work of Akahori and Takàcs), a corridor option admits the following payoff: for $K<T$,

$$
\left(A_{T}^{L, U}-K\right)_{+}=\left(\int_{0}^{T} \mathbf{1}_{\left\{h<X_{s}<H\right\}} \mathrm{d} s-K\right)_{+} .
$$

If $h=-\infty$, it is called a hurdle option. The distribution of this occupation time is linked to Lévy's arc-sine law in the BSM model. Again, expressions for the price of double-barrier corridor options are available in the BSM model and for single-barrier barrier options in Kou's model.

Miura [15] introduced $\alpha$-quantile options as an extension of lookback options. The $\alpha$-quantile of the $\log$-return process $X$ is defined, for $0 \leq \alpha \leq 1$, by

$$
q(\alpha, T):=\inf \left\{h: \int_{0}^{T} \mathbf{1}_{\left\{X_{t} \leq h\right\}} \mathrm{d} t>\alpha T\right\} .
$$

A fixed-strike $\alpha$-quantile call option admits the following payoff:

$$
\left(S_{0} \mathrm{e}^{\gamma q(\alpha, T)}-K\right)_{+}
$$

When $\alpha=0$ and $\gamma=1$, the quantile option is reduced to a lookback option. Indeed, when $\alpha=0$,

$$
q(0, T)=\sup _{0 \leq t \leq T} X_{t}
$$

In summary, in order to price many of these options, we are interested in the joint distribution of

$$
\left(\int_{0}^{T} \mathbf{1}_{\left\{L<S_{t}<U\right\}} \mathrm{d} t, S_{T}\right)
$$

or equivalently,

$$
\left(\int_{0}^{T} \mathbf{1}_{\left\{h<X_{t}<H\right\}} \mathrm{d} t, X_{T}\right)
$$

where $h=\ln \left(L / S_{0}\right)$ and $H=\ln \left(U / S_{0}\right)$.

In Black-Scholes-Merton model, in the constant elasticity of variance (CEV) model and in Kou's model, the standard technique for deriving this joint distribution (joint Laplace transform) has been to use the Feynman-Kač formula; see [9], [12] and [2].

Our goal is to price occupation-time options. In doing so, we extend results previously obtained in a nice paper by Cai, Chen and Wan [2]. We extend their results in two directions: by looking 
simultaneously at more general functionals of occupation times and a more general jump-diffusion process. For example, in order to price double step options, we derive the joint distribution of

$$
\left(\int_{0}^{T} \mathbf{1}_{\left\{X_{t}<h\right\}} \mathrm{d} t, \int_{0}^{T} \mathbf{1}_{\left\{X_{t}>H\right\}} \mathrm{d} t, X_{T}\right) .
$$

We develop a probabilistic approach to obtain these distributions in a mixed-exponential jumpdiffusion model (MEM), an approach often refered to as the perturbation approach and which is in the spirit of Géman \& Yor [8]; it is based on a decomposition of the trajectories of the underlying (log-return) process using the solutions to the one-sided and the two-sided exit problems. Our methodology uses extensions of results developed by Cai and Kou [3] (see also [5]). Finally, we answer several open questions from [2]; see e.g. the first paragraph on p. 434 and our Lemma 3.1 .

In summary, the contributions of this paper consist in new probabilistic derivations of several joint Laplace(-Carson) transforms of a mixed-exponential jump-diffusion process and its occupation times of an interval, all sampled at a fixed time, and the pricing of occupation-time derivatives such as (double-barrier) step options and $\alpha$-quantile options in a mixed-exponential model. The objectives are as in [2], but in a more general model and with a different methodology.

The rest of the paper is organized as follows. Section 2 introduces the mixed-exponential jump-diffusion process and some of its elementary properties. In Section 3, we present our main theoretical results on occupation times involving the mixed-exponential jump-diffusion process; the proofs are left for the Appendix. Finally, in Section 4, we use the results of Section 3 to derive Laplace transforms of the price for various occupation-time options.

\section{The MIXED-EXPONENTIAL JUMP-DIFFUSION PROCESS}

A Lévy jump-diffusion process $X=\left\{X_{t}, t \geq 0\right\}$ is defined as

$$
X_{t}=X_{0}+\mu t+\sigma W_{t}+\sum_{i=1}^{N_{t}} Y_{i}
$$

where $\mu \in \mathbb{R}$ and $\sigma \geq 0$ represent the drift and volatility of the diffusion part respectively, $W=\left\{W_{t}, t \geq 0\right\}$ is a (standard) Brownian motion, $N=\left\{N_{t}, t \geq 0\right\}$ is a homogeneous Poisson process with rate $\lambda$ and $\left\{Y_{i}, i=1,2, \ldots\right\}$ are independent and identically distributed random variables. These quantities are mutually independent. When $\sigma>0$, the infinitesimal generator of $X$ acts on functions $h \in \mathcal{C}_{0}^{2}(\mathbb{R})$ and is given by

$$
\mathcal{L} h(x)=\mu h^{\prime}(x)+\frac{\sigma^{2}}{2} h^{\prime \prime}(x)+\lambda \int_{-\infty}^{\infty}(h(x+y)-h(x)) f_{Y}(y) \mathrm{d} y .
$$

When $\sigma=0$, the function $h$ needs only to be once differentiable.

In a jump-diffusion market model, the dynamic of the asset price $S$ is given under the riskneutral measure $\mathbb{P}$ by:

$$
\frac{\mathrm{d} S_{t}}{S_{t-}}=r \mathrm{~d} t+\sigma \mathrm{d} W_{t}+\mathrm{d}\left(\sum_{i=1}^{N_{t}}\left(\mathrm{e}^{Y_{i}}-1\right)\right),
$$

where $r>0$ is the risk-free rate. Solving this stochastic differential equation, one obtains

$$
S_{t}=S_{0} \mathrm{e}^{X_{t}}=S_{0} \exp \left\{\mu t+\sigma W_{t}+\sum_{i=1}^{N_{t}} Y_{i}\right\},
$$


where $\mu=r-\sigma^{2} / 2-\lambda\left(\mathbb{E}\left[\mathrm{e}^{Y_{1}}\right]-1\right)$. Clearly, for that purpose, we will need to assume that the $Y_{i}$ 's have a finite moment generating function.

In the pioneer work of Merton [14, the common distribution of the $Y_{i}$ 's is chosen to be a normal distribution, while in [11] it is a double-exponential distribution, i.e. the common probability density function (pdf) is given by

$$
f_{Y}(y)=p \eta \mathrm{e}^{-\eta y} \mathbf{1}_{\{y \geq 0\}}+(1-p) \theta \mathrm{e}^{\theta y} \mathbf{1}_{\{y<0\}}
$$

where $0<p<1, \eta>0$ and $\theta>0$. When the jumps sizes are hyper-exponentially distributed, their common pdf is given by

$$
f_{Y}(y)=\sum_{i=1}^{m} p_{i} \eta_{i} \mathrm{e}^{-\eta_{i} y} \mathbf{1}_{\{y \geq 0\}}+\sum_{j=1}^{n} q_{j} \theta_{j} \mathrm{e}^{\theta_{j} y} \mathbf{1}_{\{y<0\}},
$$

where $p_{i}, q_{j}>0$ for all $i=1,2, \ldots, m$ and $j=1,2, \ldots, n$, and such that $\sum_{i=1}^{m} p_{i}+\sum_{j=1}^{n} q_{i}=1$, and where $\eta_{1}<\ldots<\eta_{m}$ and $\theta_{1}<\ldots<\theta_{n}$, then $X$ is said to be a hyper-exponential jumpdiffusion (HEJD) process and the market model is called the hyper-exponential model (HEM).

We will use a slightly more general, and thus more flexible, jump distribution: the mixedexponential distribution. In this case, the common pdf is given by

$$
f_{Y}(y)=p_{u} \sum_{i=1}^{m} p_{i} \eta_{i} \mathrm{e}^{-\eta_{i} y} \mathbf{1}_{\{y \geq 0\}}+q_{d} \sum_{j=1}^{n} q_{j} \theta_{j} \mathrm{e}^{\theta_{j} y} \mathbf{1}_{\{y<0\}},
$$

where $p_{u}, q_{d} \geq 0$ and $p_{u}+q_{d}=1$, where now $p_{i}, q_{j} \in(-\infty, \infty)$ for all $i=1,2, \ldots, m$ and $j=1,2, \ldots, n$, such that $\sum_{i=1}^{m} p_{i}=1, \sum_{j=1}^{n} q_{i}=1$, and where again $\eta_{1}<\ldots<\eta_{m}$ and $\theta_{1}<\ldots<\theta_{n}$. The resulting jump-diffusion process $X$ is said to be a mixed-exponential jumpdiffusion (MEJD) process and the market model is called the mixed-exponential model (MEM).

The MEM is a financial model fitting the data quite well and still being very tractable; for more information, see [3]. One of the main feature is probably that the mixed-exponential distribution can approximate any jump distribution (in the sense of weak convergence). See the paper by Cai and Kou [3] for more information on this process.

Throughout the rest of the paper, the law of $X$ such that $X_{0}=x$ is denoted by $\mathbb{P}_{x}$ and the corresponding expectation by $\mathbb{E}_{x}$; we write $\mathbb{P}$ and $\mathbb{E}$ when $x=0$. The Lévy exponent of a MEJD $X$ is given by

$$
\begin{aligned}
G(\zeta) & =\frac{\ln \mathbb{E}\left[\exp \left(\zeta X_{t}\right)\right]}{t} \\
& =\mu \zeta+\frac{\sigma^{2}}{2} \zeta^{2}+\lambda\left(p_{u} \sum_{i=1}^{m} \frac{p_{i} \eta_{i}}{\eta_{i}-\zeta}+q_{d} \sum_{i=1}^{n} \frac{q_{i} \theta_{i}}{\theta_{i}+\zeta}-1\right),
\end{aligned}
$$

for any $\zeta \in\left(-\theta_{1}, \eta_{1}\right)$. Then, clearly, the trend of the process is given by

$$
\mathbb{E}\left[X_{1}\right]=G^{\prime}(0+)=\mu+\lambda\left(p_{u} \sum_{i=1}^{m} \frac{p_{i}}{\eta_{i}}-q_{d} \sum_{j=1}^{m} \frac{q_{j}}{\theta_{j}}\right) .
$$

For any $\alpha \in \mathbb{R}$, the function $\zeta \mapsto G(\zeta)-\alpha$ has at most $n+m+2$ real roots. It can be shown (see [3, Theorem 3.1]) that, for a sufficiently large $\alpha>0$, the corresponding Cramér-Lundberg 
equation $G(\zeta)=\alpha$ has exactly $n+m+2$ distinct real roots; there are $m+1$ positive roots denoted by $\beta_{1, \alpha}, \ldots, \beta_{m+1, \alpha}$ and $n+1$ negative roots $\gamma_{1, \alpha}, \ldots, \gamma_{n+1, \alpha}$, satisfying

$$
\begin{gathered}
0<\beta_{1, \alpha}<\eta_{1}<\beta_{2, \alpha}<\ldots<\eta_{m}<\beta_{m+1, \alpha}<\infty, \\
-\infty<\gamma_{n+1, \alpha}<-\theta_{n}<\gamma_{n, \alpha}<\ldots<\gamma_{2, \alpha}<\theta_{1}<\gamma_{1, \alpha}<0 .
\end{gathered}
$$

Finally, let $S=m+n+2$ and define $\overrightarrow{\rho_{\alpha}}=\left(\rho_{1, \alpha}, \ldots, \rho_{S, \alpha}\right)=\left(\beta_{1, \alpha}, \ldots, \beta_{m+1, \alpha}, \gamma_{1, \alpha}, \ldots, \gamma_{n+1, \alpha}\right)$, the vector containing all the roots.

Assumption 2.1. For the rest of the paper, we assume that, for a given value of $\alpha$, the CramérLundberg equation $G(\zeta)=\alpha$ has exactly $n+m+2$ distinct real solutions as above.

Remark 2.1. In the case of a HEJD, a detailed study of the roots is undertaken in [1].

2.1. First passage and two-sided exit problems. For $b \in \mathbb{R}$, define the first passage times $\tau_{b}^{+}=\inf \left\{t \geq 0: X_{t}>b\right\}$ and $\tau_{b}^{-}=\inf \left\{t \geq 0: X_{t}<b\right\}$, with the convention inf $\emptyset=\infty$.

Consider two barrier levels $h$ and $H$ such that $h<H$. It has been shown in [3, Theorem 3.3] that, for any sufficiently large $\alpha>0, \theta<\eta_{1}$ and $x<h$,

$$
\mathbb{E}_{x}\left[\mathrm{e}^{-\alpha \tau_{h}^{+}+\theta X_{\tau_{h}^{+}}}\right]=\sum_{i=1}^{m+1} c_{i} \mathrm{e}^{\beta_{i, \alpha} x},
$$

where $\left(c_{1}, c_{2}, \ldots, c_{m+1}\right)$ is a vector of constants (uniquely determined by a nonsingular linear system). It should be pointed out here that the coefficients depend on $\alpha, \theta$ and $h$, as well as the parameters of the process (explicitly and implicitly).

Recently, in [5, Theorem 2.5], a very similar result using the same method of proof as in [3. Theorem 3.3] has been obtained for the case of a HEJD (not a MEJD) process. It has been shown that, for $\alpha \geq 0$ (and for $\sigma>0$ ), a nonnegative bounded function $g(\cdot)$ on $(h, H)^{c}$ and $h<x<H$,

$$
\mathbb{E}_{x}\left[\mathrm{e}^{-\alpha\left(\tau_{H}^{+} \wedge \tau_{h}^{-}\right)} g\left(X_{\tau_{H}^{+} \wedge \tau_{h}^{-}}\right)\right]=\sum_{i=1}^{S} b_{i} \mathrm{e}^{\rho_{i} x},
$$

where $\left(b_{1}, b_{2}, \ldots, b_{S}\right)$ is a vector of constants also to be determined.

We now provide slight extensions of the abovementioned results. The proof is left to the reader; it follows the same steps as in the proof of [3, Theorem 3.3] and [5, Theorem 2.5].

Theorem 1. Let $X$ be a MEJD process. Under Assumption 2.1 for a given value of $\alpha>0$, and for a nonnegative and bounded real-valued function $g(\cdot)$, we have:

(1) for $x<H$,

$$
\mathbb{E}_{x}\left[\mathrm{e}^{-\alpha \tau_{H}^{+}} g\left(X_{\tau_{H}^{+}}\right)\right]=\sum_{i=1}^{m+1} \omega_{i} \mathrm{e}^{\beta_{i, \alpha} x},
$$

where $\vec{\omega}=\left(\omega_{1}, \omega_{2}, \ldots, \omega_{m+1}\right)$ is a vector (uniquely) determined by the following linear system:

$$
A^{H, \alpha} \vec{\omega}=J^{H, g},
$$


where $A^{H, \alpha}$ is an $(m+1) \times(m+1)$ nonsingular matrix given by

$$
A^{H, \alpha}=\left(\begin{array}{cccc}
\mathrm{e}^{\beta_{1, \alpha} H} & \mathrm{e}^{\beta_{2, \alpha} H} & \cdots & \mathrm{e}^{\beta_{m+1, \alpha} H} \\
\frac{\mathrm{e}^{\beta_{1, \alpha} H}}{\eta_{1}-\beta_{1, \alpha}} & \frac{\mathrm{e}^{\beta_{2, \alpha} H}}{\eta_{1}-\beta_{2, \alpha}} & \cdots & \frac{\mathrm{e}^{\beta_{m+1, \alpha} H}}{\eta_{1}-\beta_{m+1, \alpha}} \\
\frac{\mathrm{e}^{\beta_{1, \alpha} H}}{\eta_{2}-\beta_{1, \alpha}} & \frac{\mathrm{e}^{\beta_{2, \alpha} H}}{\eta_{2}-\beta_{2, \alpha}} & \cdots & \frac{\mathrm{e}^{\beta_{m+1, \alpha} H}}{\eta_{2}-\beta_{m+1, \alpha}} \\
\vdots & \vdots & \ddots & \vdots \\
\frac{\mathrm{e}^{\beta_{1, \alpha} H}}{\eta_{m}-\beta_{1, \alpha}} & \frac{\mathrm{e}^{\beta_{2, \alpha} H}}{\eta_{m}-\beta_{2, \alpha}} & \cdots & \frac{\mathrm{e}^{\beta_{m+1, \alpha} H}}{\eta_{m}-\beta_{m+1, \alpha}}
\end{array}\right),
$$

and where $J^{H, g}$ is an $(m+1)$-dimensional vector given by

$$
\left(g(H+), \mathrm{e}^{\eta_{1} H} \int_{H}^{\infty} g(y) \mathrm{e}^{-\eta_{1} y} \mathrm{~d} y, \mathrm{e}^{\eta_{2} H} \int_{H}^{\infty} g(y) \mathrm{e}^{-\eta_{2} y} \mathrm{~d} y, \ldots, \mathrm{e}^{\eta_{m} H} \int_{H}^{\infty} g(y) \mathrm{e}^{-\eta_{m} y} \mathrm{~d} y\right) .
$$

(2) for $x>h$,

$$
\mathbb{E}_{x}\left[\mathrm{e}^{-\alpha \tau_{h}^{-}} g\left(X_{\tau_{h}^{-}}\right)\right]=\sum_{i=1}^{n+1} \nu_{i} \mathrm{e}^{\gamma_{i, \alpha} x}
$$

where $\vec{\nu}=\left(\nu_{1}, \nu_{2}, \ldots, \nu_{n+1}\right)$ is a vector (uniquely) determined by the following linear system:

$$
A^{h, \alpha} \vec{\nu}=J^{h, g}
$$

where $A^{h, \alpha}$ is an $(n+1) \times(n+1)$ nonsingular matrix given by

$$
A^{h, \alpha}=\left(\begin{array}{cccc}
\mathrm{e}^{\gamma_{1, \alpha} h} & \mathrm{e}^{\gamma_{2, \alpha} h} & \cdots & \mathrm{e}^{\gamma_{n+1, \alpha} h} \\
\frac{\mathrm{e}^{\gamma_{1, \alpha} h}}{\theta_{1}+\gamma_{1, \alpha}} & \frac{\mathrm{e}^{\gamma_{2, \alpha} h}}{\theta_{1}+\gamma_{2, \alpha}} & \cdots & \frac{\mathrm{e}^{\gamma_{n+1, \alpha} h}}{\theta_{1}+\gamma_{n+1, \alpha}} \\
\frac{\mathrm{e}^{\gamma_{1, \alpha} h}}{\theta_{2}+\gamma_{1, \alpha}} & \frac{\mathrm{e}^{\gamma_{2}, \alpha}}{\theta_{2}+\gamma_{2, \alpha}} & \cdots & \frac{\mathrm{e}^{\gamma_{n+1, \alpha} h}}{\theta_{2}+\gamma_{n+1, \alpha}} \\
\vdots & \vdots & \ddots & \vdots \\
\frac{\mathrm{e}^{\gamma_{1, \alpha} h}}{\theta_{n}+\gamma_{1, \alpha}} & \frac{\mathrm{e}^{\gamma_{2, \alpha} h}}{\theta_{n}+\gamma_{2, \alpha}} & \cdots & \frac{\mathrm{e}^{\gamma_{n+1, \alpha} h}}{\theta_{n}+\gamma_{n+1, \alpha}}
\end{array}\right),
$$

and where $J^{h, g}$ is an $(n+1)$-dimensional vector given by

$$
\left(g(h-), \mathrm{e}^{-\theta_{1} h} \int_{-\infty}^{h} g(y) \mathrm{e}^{\theta_{1} y} \mathrm{~d} y, \mathrm{e}^{-\theta_{2} h} \int_{-\infty}^{h} g(y) \mathrm{e}^{\theta_{2} y} \mathrm{~d} y, \ldots, \mathrm{e}^{-\theta_{n} h} \int_{-\infty}^{h} g(y) \mathrm{e}^{\theta_{n} y} \mathrm{~d} y\right) .
$$

(3) for $h<x<H$,

$$
\mathbb{E}_{x}\left[\mathrm{e}^{-\alpha\left(\tau_{H}^{+} \wedge \tau_{h}^{-}\right) g}\left(X_{\tau_{H}^{+} \wedge \tau_{h}^{-}}\right)\right]=\sum_{i=1}^{m+1} \omega_{i} \mathrm{e}^{\beta_{i, \alpha} x}+\sum_{i=1}^{n+1} \nu_{i} \mathrm{e}^{\gamma_{i, \alpha} x}=\sum_{i=1}^{S} Q_{i} \mathrm{e}^{\rho_{i, \alpha} x}
$$

where $\mathbf{Q}=\left(Q_{1}, Q_{2}, \ldots, Q_{S}\right)=\left(\omega_{1}, \omega_{2}, \ldots, \omega_{m+1}, \nu_{1}, \nu_{2}, \ldots, \nu_{n+1}\right)$ is a vector (uniquely) determined by the following linear system:

$$
A^{h, H, \alpha} \mathbf{Q}=J^{h, H, g},
$$


where $A^{h, H, \alpha}$ is an $S \times S$ nonsingular matrix given by

$$
\begin{aligned}
& A^{h, H, \alpha}=\left(\begin{array}{cccccc}
\mathrm{e}^{\beta_{1, \alpha} h} & \ldots & \mathrm{e}^{\beta_{m+1, \alpha} h} & \mathrm{e}^{\gamma_{1, \alpha} h} & \ldots & \mathrm{e}^{\gamma_{n+1, \alpha} h} \\
\mathrm{e}^{\beta_{1, \alpha} H} & \cdots & \mathrm{e}^{\beta_{m+1, \alpha} H} & \mathrm{e}^{\gamma_{1, \alpha} H} & \cdots & \mathrm{e}^{\gamma_{n+1, \alpha} H} \\
\frac{\mathrm{e}^{\beta_{1, \alpha} h}}{\theta_{1}+\beta_{1, \alpha}} & \cdots & \frac{\mathrm{e}^{\beta_{m+1, \alpha} h}}{\theta_{1}+\beta_{m+1, \alpha}} & \frac{\mathrm{e}^{\gamma_{1, \alpha} h}}{\theta_{1}+\gamma_{1, \alpha}} & \cdots & \frac{\mathrm{e}^{\gamma_{n+1, \alpha} h}}{\theta_{1}+\gamma_{n+1, \alpha}} \\
\vdots & \vdots & \ddots & \vdots & \vdots & \\
\frac{\mathrm{e}^{\beta_{1, \alpha} h}}{\theta_{n}+\beta_{1, \alpha}} & \cdots & \frac{\mathrm{e}^{\beta_{m+1, \alpha} h}}{\theta_{n}+\beta_{m+1, \alpha}} & \frac{\mathrm{e}^{\gamma_{1, \alpha} h}}{\theta_{n}+\gamma_{1, \alpha}} & \cdots & \frac{\mathrm{e}^{\gamma_{n+1, \alpha} h}}{\theta_{n}+\gamma_{n+1, \alpha}} \\
\frac{\mathrm{e}^{\beta_{1, \alpha} H}}{\eta_{1}-\beta_{1, \alpha}} & \cdots & \frac{\mathrm{e}^{\beta_{m+1, \alpha} H}}{\eta_{1}-\beta_{m+1, \alpha}} & \frac{\mathrm{e}^{\gamma_{1, \alpha} H}}{\eta_{1}-\gamma_{1, \alpha}} & \cdots & \frac{\mathrm{e}^{\gamma_{n+1, \alpha} H}}{\eta_{1}-\gamma_{n+1, \alpha}} \\
\vdots & \vdots & \ddots & \vdots & \vdots & \\
\frac{\mathrm{e}^{\beta_{1, \alpha} H}}{\eta_{m}-\beta_{1, \alpha}} & \cdots & \frac{\mathrm{e}^{\beta_{m+1, \alpha} H}}{\eta_{m}-\beta_{m+1, \alpha}} & \frac{\mathrm{e}^{\gamma_{1, \alpha} H}}{\eta_{m}-\gamma_{1, \alpha}} & \cdots & \frac{\mathrm{e}^{\gamma_{n+1, \alpha} H}}{\eta_{m}-\gamma_{n+1, \alpha}}
\end{array}\right) \\
& =\left(\begin{array}{cccc}
\mathrm{e}^{\rho_{1, \alpha} h} & \mathrm{e}^{\rho_{2, \alpha} h} & \cdots & \mathrm{e}^{\rho_{S, \alpha} h} \\
\mathrm{e}^{\rho_{1, \alpha} H} & \mathrm{e}^{\rho_{2, \alpha} H} & \cdots & \mathrm{e}^{\rho_{S, \alpha} H} \\
\frac{\mathrm{e}^{\rho_{1, \alpha} h}}{\theta_{1}+\rho_{1, \alpha}} & \frac{\mathrm{e}^{\rho_{2, \alpha} h}}{\theta_{1}+\rho_{2, \alpha}} & \cdots & \frac{\mathrm{e}^{\rho_{S, \alpha} h}}{\theta_{1}+\rho_{S, \alpha}} \\
\vdots & \vdots & \ddots & \vdots \\
\frac{\mathrm{e}^{\rho_{1, \alpha} h}}{\theta_{n}+\rho_{1, \alpha}} & \frac{\mathrm{e}^{\rho_{2, \alpha} h}}{\theta_{n}+\rho_{2, \alpha}} & \cdots & \frac{\mathrm{e}^{\rho_{S, \alpha} h}}{\theta_{n}+\rho_{S, \alpha}} \\
\frac{\mathrm{e}^{\rho_{1, \alpha} H}}{\eta_{1}-\rho_{1, \alpha}} & \frac{\mathrm{e}^{\rho_{2, \alpha} H}}{\eta_{1}-\rho_{2, \alpha}} & \cdots & \frac{\mathrm{e}^{\rho_{S, \alpha} H}}{\eta_{1}-\rho_{S, \alpha}} \\
\vdots & \vdots & \ddots & \vdots \\
\frac{\mathrm{e}^{\rho_{1, \alpha} H}}{\eta_{m}-\rho_{1, \alpha}} & \frac{\mathrm{e}^{\rho_{2, \alpha} H}}{\eta_{m}-\rho_{2, \alpha}} & \cdots & \frac{\mathrm{e}^{\rho_{S, \alpha} H}}{\eta_{m}-\rho_{S, \alpha}}
\end{array}\right)
\end{aligned}
$$

and where $J^{h, H, g}$ is an $S$-dimensional vector given by

$$
J^{h, H, g}=\left(J^{H, g}, J^{h, g}\right) .
$$

Clearly, by the definition of the stopping times, if $x>h$ (resp. $x<H$ ), then

$$
\mathbb{E}_{x}\left[\mathrm{e}^{-\alpha \tau_{h}^{+}} g\left(X_{\tau_{h}^{+}}\right)\right]=g(x) \quad\left(\text { resp. } \quad \mathbb{E}_{x}\left[\mathrm{e}^{-\alpha \tau_{H}^{-}} g\left(X_{\tau_{H}^{-}}\right)\right]=g(x)\right),
$$

and, if $x<h$ or $x>H$, then

$$
\mathbb{E}_{x}\left[\mathrm{e}^{-\alpha\left(\tau_{H}^{+} \wedge \tau_{h}^{-}\right)} g\left(X_{\tau_{H}^{+} \wedge \tau_{h}^{-}}\right)\right]=g(x) .
$$

\section{Our main Results}

Our first objective is to obtain the joint distribution of

$$
\left(\int_{0}^{T} \mathbf{1}_{\left\{h<X_{t}<H\right\}} \mathrm{d} t, X_{T}\right)
$$

for a given $T>0$. In order to do so, we will compute the following joint Laplace-Carson transform with respect to $T$ : for each $x \in \mathbb{R}$, set

$$
w(x ; h, H, \alpha, \rho, \gamma):=\int_{0}^{\infty} \alpha \mathrm{e}^{-\alpha T} \mathbb{E}_{x}\left[\mathrm{e}^{-\rho \int_{0}^{T} \mathbf{1}_{\left\{h<X_{t}<H\right\}} \mathrm{d} t+\gamma X_{T}}\right] \mathrm{d} T,
$$


where $\alpha>0, \rho \geq 0$ and $\gamma \in \mathbb{R}$. Clearly, we have

$$
w(x)=\mathbb{E}_{x}\left[\mathrm{e}^{-\rho \int_{0}^{\mathbf{e}_{\alpha}} \mathbf{1}_{\left\{h<X_{t}<H\right\}} \mathrm{d} t+\gamma X_{\mathbf{e}_{\alpha}}}\right],
$$

where $\mathbf{e}_{\alpha}$ is an exponentially distributed random variable (independent of $X$ ) with mean $1 / \alpha$.

Here is our main result.

Theorem 2. For any $0 \leq \gamma<\min \left(\eta_{1}, \theta_{1}\right), \rho>0$ and $G(\gamma)<\alpha$, we have

$$
\begin{aligned}
\int_{0}^{\infty} \alpha \mathrm{e}^{-\alpha T} \mathbb{E}_{x}\left[\mathrm{e}^{\left.-\rho \int_{0}^{T} \mathbf{1}_{\left\{h<X_{t}<H\right\}} \mathrm{d} t+\gamma X_{T}\right] \mathrm{d} T}\right. \\
= \begin{cases}\sum_{i=1}^{m+1} \omega_{i}^{L} \mathrm{e}^{\beta_{i, \alpha}(x-h)}-c_{L} \mathrm{e}^{\gamma x}, & x \leq h, \\
-\sum_{i=1}^{m+1} \omega_{i}^{0} \mathrm{e}^{\beta_{i, \alpha+\rho}(x-H)}-\sum_{j=1}^{n+1} \nu_{j}^{0} \mathrm{e}^{-\gamma_{j, \alpha+\rho}(x-h)}-c_{0} \mathrm{e}^{\gamma x}, & h<x<H, \\
\sum_{j=1}^{n+1} \nu_{j}^{U} \mathrm{e}^{-\gamma_{j, \alpha}(x-H)}-c_{U} \mathrm{e}^{\gamma x}, & x \geq H,\end{cases}
\end{aligned}
$$

where

$$
c_{L}=c_{U}=\frac{\alpha}{G(\gamma)-\alpha}, \quad c_{0}=\frac{\alpha}{G(\gamma)-(\alpha+\rho)} .
$$

The vector of coefficients

$$
Q=\left(\omega_{i}^{L} ; \omega_{i}^{0} ; \nu_{j}^{0} ; \nu_{j}^{U} ; i=1, \ldots, m+1 ; j=1, \ldots, n+1\right)
$$

satisfies a linear system

$$
B Q=V
$$

Here $V$ is a $2 S$-dimensional vector,

$$
V=\left(c_{U}-c_{0}\right)\left(\begin{array}{l}
V_{1} \\
V_{2}
\end{array}\right)
$$

where the $S$-dimensional column vectors $V_{1}$ and $V_{2}$ are given by

$$
\begin{aligned}
& V_{1}=\left(\mathrm{e}^{\gamma h}, \gamma \mathrm{e}^{\gamma h}, \frac{\mathrm{e}^{\gamma h}}{\eta_{1}-\gamma}, \cdots, \frac{\mathrm{e}^{\gamma h}}{\eta_{m}-\gamma}, \frac{\mathrm{e}^{\gamma h}}{\theta_{1}+\gamma}, \cdots, \frac{\mathrm{e}^{\gamma h}}{\theta_{n}+\gamma}\right) \\
& V_{2}=\left(\mathrm{e}^{\gamma H}, \gamma \mathrm{e}^{\gamma H}, \frac{\mathrm{e}^{\gamma H}}{\eta_{1}-\gamma}, \cdots, \frac{\mathrm{e}^{\gamma H}}{\eta_{m}-\gamma}, \frac{\mathrm{e}^{\gamma H}}{\theta_{1}+\gamma}, \cdots, \frac{\mathrm{e}^{\gamma H}}{\theta_{n}+\gamma}\right)
\end{aligned}
$$

and $B$ is a $2 S \times 2 S$ matrix

$$
B=\left(\begin{array}{cc}
M & N Z_{\beta} \\
M Z_{\gamma} & N
\end{array}\right)
$$

where $Z_{\beta}$ and $Z_{\gamma}$ are $S \times S$ diagonal matrices with elements

$$
\left\{0, \ldots, 0, \mathrm{e}^{\beta_{1, \alpha+\rho}(h-H)}, \ldots, \mathrm{e}^{\beta_{m+1, \alpha+\rho}(h-H)}\right\}
$$

and

$$
\left\{0, \ldots, 0, \mathrm{e}^{\gamma_{1, \alpha+\rho}(h-H)}, \ldots, \mathrm{e}^{\gamma_{n+1, \alpha+\rho}(h-H)}\right\}
$$


respectively, and where $M$ and $N$ are given by

$$
M=\left(\begin{array}{cccccc}
1 & \cdots & 1 & 1 & \cdots & 1 \\
\beta_{1, \alpha} & \cdots & \beta_{m+1, \alpha} & -\gamma_{1, \alpha+\rho} & \cdots & -\gamma_{n+1, \alpha+\rho} \\
\frac{1}{\eta_{1}-\beta_{1, \alpha}} & \cdots & \frac{1}{\eta_{1}-\beta_{m+1, \alpha}} & \frac{1}{\eta_{1}+\gamma_{1, \alpha+\rho}} & \cdots & \frac{1}{\eta_{1}+\gamma_{n+1, \alpha+\rho}} \\
\vdots & \ddots & \vdots & \vdots & \ddots & \vdots \\
\frac{1}{\eta_{m}-\beta_{1, \alpha}} & \cdots & \frac{1}{\eta_{m}-\beta_{m+1, \alpha}} & \frac{1}{\eta_{m}+\gamma_{1, \alpha+\rho}} & \cdots & \frac{1}{\eta_{m}+\gamma_{n+1, \alpha+\rho}} \\
\frac{1}{\theta_{1}+\beta_{1, \alpha}} & \cdots & \frac{1}{\theta_{1}+\beta_{m+1, \alpha}} & \frac{1}{\theta_{1}-\gamma_{1, \alpha+\rho}} & \cdots & \frac{1}{\theta_{1}-\gamma_{n+1, \alpha+\rho}} \\
\vdots & \ddots & \vdots & \vdots & \ddots & \vdots \\
\frac{1}{\theta_{n}+\beta_{1, \alpha}} & \cdots & \frac{1}{\theta_{n}+\beta_{m+1, \alpha}} & \frac{1}{\theta_{n}-\gamma_{1, \alpha+\rho}} & \cdots & \frac{1}{\theta_{n}-\gamma_{n+1, \alpha+\rho}}
\end{array}\right)
$$

and

$$
N=\left(\begin{array}{cccccc}
1 & \cdots & 1 & 1 & \cdots & 1 \\
-\gamma_{1, \alpha} & \cdots & -\gamma_{n+1, \alpha} & \beta_{1, \alpha+\rho} & \cdots & \beta_{m+1, \alpha+\rho} \\
\frac{1}{\eta_{1}+\gamma_{1, \alpha}} & \cdots & \frac{1}{\eta_{1}+\gamma_{n+1, \alpha}} & \frac{1}{\eta_{1}-\beta_{1, \alpha+\rho}} & \cdots & \frac{1}{\eta_{1}-\beta_{m+1, \alpha+\rho}} \\
\vdots & \ddots & \vdots & \vdots & \ddots & \vdots \\
\frac{1}{\eta_{m}+\gamma_{1, \alpha}} & \cdots & \frac{1}{\eta_{n}+\gamma_{n+1, \alpha}} & \frac{1}{\eta_{m}-\beta_{1, \alpha+\rho}} & \cdots & \frac{1}{\eta_{m}-\beta_{m+1, \alpha+\rho}} \\
\frac{1}{\theta_{1}-\gamma_{1, \alpha}} & \cdots & \frac{1}{\theta_{1}-\gamma_{n+1, \alpha}} & \frac{1}{\theta_{1}+\beta_{1, \alpha+\rho}} & \cdots & \frac{1}{\theta_{1}+\beta_{m+1, \alpha+\rho}} \\
\vdots & \ddots & \vdots & \vdots & \ddots & \vdots \\
\frac{1}{\theta_{n}-\gamma_{1, \alpha}} & \cdots & \frac{1}{\theta_{n}-\gamma_{n+1, \alpha}} & \frac{1}{\theta_{n}+\beta_{1, \alpha+\rho}} & \cdots & \frac{1}{\theta_{n}+\beta_{m+1, \alpha+\rho}}
\end{array}\right) .
$$

In order for the last Theorem to yield an explicit result, we must show that the linear system in (5) is solvable:

Lemma 3.1. Under Assumption [2.1, for a given value of $\alpha>0$, the matrix $B$ given in (7) is invertible.

Proof. Let $S=m+n+2$ and assume that $B C=0$ for some vector $C=\left(C_{1}, C_{2}, \ldots, C_{2 S}\right)$. Consider the function $V(x)=\sum_{i=1}^{2 S} C_{i} \mathrm{e}^{\rho_{i} x}$ for $x \in(h, H)$, and $V(x)=0$ otherwise, with $\rho_{1}, \ldots, \rho_{2 S}$ be the distinct real zeros of the equation $G(x)=\alpha$. Since $B C=0$ and $V(x)$ is a solution to the boundary value problem

$$
\begin{cases}\left(\mathcal{L}-\alpha-\rho \mathbf{1}_{\{h<x<H\}}\right) \phi(x)=0 & x \in(h, H), \\ \phi(x)=0 & x \in(-\infty, h] \cup[H,+\infty) .\end{cases}
$$

From the uniqueness of the solution to the boundary value problem (8), $V(x) \equiv 0$ on $(h, H)$. Now, since $\left\{\mathrm{e}^{\rho_{i} x}, \leq i \leq 2 S\right\}$ are linearly independent then $C=0$ and $B$ is invertible.

Using the same methodology as in the proof of Theorem 2 , we can prove the following result. 
Theorem 3. For any $0 \leq \gamma<\min \left(\eta_{1}, \theta_{1}\right), \rho_{1}>0, \rho_{2}>0$ and $G(\gamma)<\alpha$, we have for $h<H$,

$$
\begin{aligned}
\int_{0}^{\infty} \alpha \mathrm{e}^{-\alpha T} \mathbb{E}_{x}\left[\mathrm{e}^{\left.-\rho_{1} \int_{0}^{T} \mathbf{1}_{\left\{X_{t} \leq h\right\}} \mathrm{d} t-\rho_{2} \int_{0}^{T} \mathbf{1}_{\left\{X_{t} \geq H\right\}} \mathrm{d} t+\gamma X_{T}\right] \mathrm{d} T}\right. \\
= \begin{cases}\sum_{i=1}^{m+1} \omega_{i}^{L} \mathrm{e}^{\beta_{i, \alpha+\rho}(x-h)}-c_{L} \mathrm{e}^{\gamma x}, & x \leq h, \\
-\sum_{i=1}^{m+1} \omega_{i}^{0} \mathrm{e}^{\beta_{i, \alpha}(x-H)}-\sum_{j=1}^{n+1} \nu_{j}^{0} \mathrm{e}^{-\gamma_{j, \alpha}(x-h)}-c_{0} \mathrm{e}^{\gamma x}, & h<x<H, \\
\sum_{j=1}^{n+1} \nu_{j}^{U} \mathrm{e}^{-\gamma_{j, a+\rho_{2}}(x-H)}-c_{U} \mathrm{e}^{\gamma x}, & x \geq H,\end{cases}
\end{aligned}
$$

where

$$
c_{L}=\frac{\alpha}{G(\gamma)-\left(\alpha+\rho_{1}\right)}, \quad c_{0}=\frac{\alpha}{G(\gamma)-\alpha}, \quad \text { and } \quad c_{U}=\frac{\alpha}{G(\gamma)-\left(\alpha+\rho_{2}\right)} .
$$

The vector of coefficients

$$
Q^{\prime}=\left(\omega_{i}^{L} ; w_{i}^{0} ; \nu_{j}^{0} ; \nu_{j}^{U} ; i=1, \ldots, m+1 ; j=1, \ldots, n+1\right)
$$

satisfies a linear system

$$
B^{\prime} Q^{\prime}=V
$$

Here $V$ is the vector defined in (6), $B^{\prime}$ is a $2 S \times 2 S$ matrix given by

$$
B^{\prime}=\left(\begin{array}{cc}
M^{\prime} & N^{\prime} Z_{\beta} \\
M^{\prime} Z_{\gamma} & N^{\prime}
\end{array}\right)
$$

where $Z_{\beta}$ and $Z_{\gamma}$ are $S \times S$ diagonal matrices with elements

$$
\left\{0, \ldots, 0, \mathrm{e}^{\beta_{1, \alpha}(h-H)}, \ldots, \mathrm{e}^{\beta_{m+1, \alpha}(h-H)}\right\}
$$

and

$$
\left\{0, \ldots, 0, \mathrm{e}^{\gamma_{1, \alpha}(h-H)}, \ldots, \mathrm{e}^{\gamma_{n+1, \alpha}(h-H)}\right\},
$$

respectively, and where $M^{\prime}$ and $N^{\prime}$ are given by

$$
M^{\prime}=\left(\begin{array}{cccccc}
1 & \cdots & 1 & 1 & \cdots & 1 \\
\beta_{1, \alpha+\rho_{1}} & \cdots & \beta_{m+1, \alpha+\rho_{1}} & -\gamma_{1, \alpha} & \cdots & -\gamma_{n+1, \alpha} \\
\frac{1}{\eta_{1}-\beta_{1, \alpha+\rho_{1}}} & \cdots & \frac{1}{\eta_{1}-\beta_{m+1, \alpha+\rho_{1}}} & \frac{1}{\eta_{1}+\gamma_{1, \alpha}} & \cdots & \frac{1}{\eta_{1}+\gamma_{n+1, \alpha}} \\
\vdots & \ddots & \vdots & \vdots & \ddots & \vdots \\
\frac{1}{\eta_{m}-\beta_{1, \alpha+\rho_{1}}} & \cdots & \frac{1}{\eta_{m}-\beta_{m+1, \alpha+\rho_{1}}} & \frac{1}{\eta_{m}+\gamma_{1, \alpha}} & \cdots & \frac{1}{\eta_{m}+\gamma_{n+1, \alpha}} \\
\frac{1}{\theta_{1}+\beta_{1, \alpha+\rho_{1}}} & \cdots & \frac{1}{\theta_{1}+\beta_{m+1, \alpha+\rho_{1}}} & \frac{1}{\theta_{1}-\gamma_{1, \alpha}} & \cdots & \frac{1}{\theta_{1}-\gamma_{n+1, \alpha}} \\
\vdots & \ddots & \vdots & \vdots & \ddots & \vdots \\
\frac{1}{\theta_{n}+\beta_{1, \alpha+\rho_{1}}} & \cdots & \frac{1}{\theta_{n}+\beta_{m+1, \alpha+\rho_{1}}} & \frac{1}{\theta_{n}-\gamma_{1, \alpha}} & \cdots & \frac{1}{\theta_{n}-\gamma_{n+1, \alpha}}
\end{array}\right)
$$


and

$$
N^{\prime}=\left(\begin{array}{cccccc}
1 & \cdots & 1 & 1 & \cdots & 1 \\
-\gamma_{1, \alpha+\rho_{2}} & \cdots & -\gamma_{n+1, \alpha+\rho_{2}} & \beta_{1, \alpha} & \cdots & \beta_{m+1, \alpha} \\
\frac{1}{\eta_{1}+\gamma_{1, \alpha+\rho_{2}}} & \cdots & \frac{1}{\eta_{1}+\gamma_{n+1, \alpha+\rho_{2}}} & \frac{1}{\eta_{1}-\beta_{1, \alpha}} & \cdots & \frac{1}{\eta_{1}-\beta_{m+1, \alpha}} \\
\vdots & \ddots & \vdots & \vdots & \ddots & \vdots \\
\frac{1}{\eta_{m}+\gamma_{1, \alpha+\rho_{2}}} & \cdots & \frac{1}{\eta_{m}+\gamma_{n+1, \alpha+\rho_{2}}} & \frac{1}{\eta_{m}-\beta_{1, \alpha}} & \cdots & \frac{1}{\eta_{m}-\beta_{m+1, \alpha}} \\
\frac{1}{\theta_{1}-\gamma_{1, \alpha+\rho_{2}}} & \cdots & \frac{1}{\theta_{1}-\gamma_{n+1, \alpha+\rho_{2}}} & \frac{1}{\theta_{1}+\beta_{1, \alpha}} & \cdots & \frac{1}{\theta_{1}+\beta_{m+1, \alpha}} \\
\vdots & \ddots & \vdots & \vdots & \ddots & \vdots \\
\frac{1}{\theta_{n}-\gamma_{1, \alpha+\rho_{2}}} & \cdots & \frac{1}{\theta_{n}-\gamma_{n+1, \alpha+\rho_{2}}} & \frac{1}{\theta_{n}+\beta_{1, \alpha}} & \cdots & \frac{1}{\theta_{n}+\beta_{m+1, \alpha}}
\end{array}\right) .
$$

In Theorem 3, if we let $H \rightarrow h$, then it greatly simplifies the expression:

Corollary 1. Given the constants $\rho_{1}>0, \rho_{2}>0, \gamma \geq 0$ and $\alpha>0$ such that $\gamma<\min \left(\eta_{1}, \theta_{1}\right)$ and $G(\gamma)<\alpha$, we have

$$
\begin{aligned}
\int_{0}^{\infty} \alpha \mathrm{e}^{-\alpha T} \mathbb{E}_{x}\left[\mathrm{e}^{-\rho_{1} \int_{0}^{T} \mathbf{1}_{\left\{X_{s} \leq h\right\} \mathrm{d} s}-\rho_{2} \int_{0}^{T} \mathbf{1}_{\left\{X_{s} \geq h\right\}}+\gamma X_{T}}\right] \mathrm{d} T \\
\quad= \begin{cases}\sum_{i=1}^{m+1} \omega_{i} \mathrm{e}^{\beta_{i, \alpha+\rho_{1}}(x-h)}-c_{1} \mathrm{e}^{\gamma x}, & x \leq h, \\
\sum_{j=1}^{n+1} \nu_{j} \mathrm{e}^{-\gamma_{j, \alpha+\rho_{2}}(x-h)}-c_{2} \mathrm{e}^{\gamma x}, & x>h,\end{cases}
\end{aligned}
$$

where

$$
c_{1}=\frac{\alpha}{G(\gamma)-\left(\alpha+\rho_{1}\right)}, \quad c_{2}=\frac{\alpha}{G(\gamma)-\left(\alpha+\rho_{2}\right)} .
$$

For $i=1, \ldots, m+1$,

$$
\omega_{i}=\frac{\prod_{j=1, j \neq i}^{m+1}\left(\beta_{j, \alpha+\rho_{1}}-\gamma\right) \prod_{k=1}^{n+1}\left(-\gamma_{k, \alpha+\rho_{2}}-\gamma\right) \prod_{j=1}^{m}\left(\eta_{j}-\beta_{i, \alpha+\rho_{1}}\right) \prod_{k=1}^{n}\left(\theta_{k}+\beta_{i, \alpha+\rho_{1}}\right)}{\prod_{j=1, j \neq i}^{m+1}\left(\beta_{j, \alpha+\rho_{1}}-\beta_{i, \alpha+\rho_{1}}\right) \prod_{k=1}^{n+1}\left(-\gamma_{k, \alpha+\rho_{2}}-\beta_{i, \alpha+\rho_{1}}\right) \prod_{j=1}^{m}\left(\eta_{j}-\gamma\right) \prod_{k=1}^{n}\left(\theta_{k}+\gamma\right)} c_{12},
$$

and, for $i=1, \ldots, n+1$,

$$
\nu_{i}=\frac{\prod_{j=1}^{m+1}\left(\beta_{j, \alpha+\rho_{1}}-\gamma\right) \prod_{k=1, k \neq i}^{n+1}\left(-\gamma_{k, \alpha+\rho_{2}}-\gamma\right) \prod_{j=1}^{m}\left(\eta_{j}+\gamma_{i, \alpha+\rho_{2}}\right) \prod_{k=1}^{n}\left(\theta_{k}-\gamma_{i, \alpha+\rho_{2}}\right)}{\prod_{j=1}^{m+1}\left(\beta_{j, \alpha+\rho_{1}}+\gamma_{i, \alpha+\rho_{2}}\right) \prod_{k=1, k \neq i}^{n+1}\left(-\gamma_{k, \alpha+\rho_{2}}+\gamma_{i, \alpha+\rho_{2}}\right) \prod_{j=1}^{m}\left(\eta_{j}-\gamma\right) \prod_{k=1}^{n}\left(\theta_{k}+\gamma\right)} c_{12},
$$

with $c_{12}=c_{1}-c_{2}$.

Proof. By Gauss elimination, we can show that the determinant of

$$
A=\left(\begin{array}{cccc}
1 & 1 & \cdots & 1 \\
a_{1} & a_{2} & \cdots & a_{S} \\
\frac{1}{\eta_{1}-a_{1}} & \frac{1}{\eta_{1}-a_{2}} & \cdots & \frac{1}{\eta_{1}-a_{S}} \\
\vdots & \vdots & \ddots & \vdots \\
\frac{1}{\eta_{m}-a_{1}} & \frac{1}{\eta_{m}-a_{2}} & \cdots & \frac{1}{\eta_{m}-a_{S}} \\
\frac{1}{\theta_{1}+a_{1}} & \frac{1}{\theta_{1}+a_{2}} & \cdots & \frac{1}{\theta_{1}+a_{S}} \\
\vdots & \vdots & \ddots & \vdots \\
\frac{1}{\theta_{n}+a_{1}} & \frac{1}{\theta_{n}+a_{2}} & \cdots & \frac{1}{\theta_{n}+a_{S}}
\end{array}\right)
$$


where $S=m+n+2$, is given by

$$
\operatorname{det}(A)=-\frac{\left(\sum_{i=1}^{m} \eta_{i}+\sum_{j=1}^{n} \theta_{j}\right) \prod_{1 \leq i<j \leq S}\left(a_{i}-a_{j}\right)}{\prod_{1 \leq i \leq S, 1 \leq j \leq S}\left(\prod_{1 \leq k \leq m}\left(\eta_{k}-a_{i}\right) \prod_{1 \leq l \leq n}\left(\theta_{l}+a_{j}\right)\right)} .
$$

If $\operatorname{det}(A) \neq 0$, then the matrix $A$ is invertible and, for the column vector

$$
\Delta=\left(1, \gamma, \frac{1}{\eta_{1}-\gamma}, \ldots, \frac{1}{\eta_{m}-\gamma}, \frac{1}{\theta_{1}+\gamma}, \ldots, \frac{1}{\theta_{n}+\gamma}\right),
$$

the linear system $A Y=\Delta$ has a unique solution $Y^{*}=\left(y_{1}^{*}, \ldots, y_{S}^{*}\right)$, where, for $i=1,2, \ldots, S$,

$$
y_{i}^{*}=\frac{\prod_{j \neq i}\left(a_{j}-\gamma\right) \prod_{k=1}^{m}\left(\eta_{k}-a_{i}\right) \prod_{l=1}^{n}\left(\theta_{k}+a_{i}\right)}{\prod_{j \neq i}\left(a_{j}-a_{i}\right) \prod_{k=1}^{m}\left(\eta_{k}-\gamma\right) \prod_{l=1}^{n}\left(\theta_{k}+\gamma\right)} .
$$

Applying the above to Theorem 3 when $H \rightarrow h$ completes the proof.

\section{OCCUPATION-TIME OPTION PRICING}

We now show how our theoretical results can be easily applied to the pricing of various occupation-time options. The idea is to obtained explicit expressions for (double) Laplace transforms of option prices, which can then be inverted using well-known and well-studied Laplace inversion techniques to get numerical prices; see e.g. [10] and [3] and the references therein. Note that using this methodology, together with the results of Section 3, many other (and more complicated) occupation-time derivatives could be analyzed.

4.1. Step and double-barrier step options. As already mentioned in the Introduction, a (down-and-out call) step option admits the following payoff:

$$
\mathrm{e}^{-\rho \int_{0}^{T} \mathbf{1}_{\left\{S_{t} \leq L\right\}} \mathrm{d} t}\left(S_{T}-K\right)^{+}=\mathrm{e}^{-\rho \int_{0}^{T} \mathbf{1}_{\left\{X_{t} \leq \ln \left(L / S_{0}\right)\right\}} \mathrm{d} t}\left(S_{0} \mathrm{e}^{X_{T}}-K\right)^{+} .
$$

Then, its price can be written as

$$
C^{\text {step }}(k, T):=\mathrm{e}^{-r T} \mathbb{E}\left[\mathrm{e}^{-\rho \int_{0}^{T} \mathbf{1}_{\left\{X_{t} \leq \ln \left(L / S_{0}\right)\right\}} \mathrm{d} t}\left(S_{0} \mathrm{e}^{X_{T}}-\mathrm{e}^{-k}\right)^{+}\right],
$$

where $k=-\ln (K)$. Following Carr and Madan's approach for vanilla options, as in [4] (see also [10] and [3]), we can easily compute the double Laplace transform of $C^{\text {step }}(k, T)$ :

$$
\int_{0}^{\infty} \int_{-\infty}^{\infty} \mathrm{e}^{-\alpha T-\beta k} C^{\text {step }}(k, T) \mathrm{d} k \mathrm{~d} T=\mathrm{e}^{-r T} \frac{S_{0}^{\beta+1} w\left(\ln \left(S_{0}\right) ;-\infty, \ln \left(L / S_{0}\right), \alpha, \rho, \beta+1\right)}{\alpha \beta(\beta+1)},
$$

where $w(x ; h, H, \alpha, \rho, \gamma)$ is given by Theorem 2 . Note that the double Laplace transform of delta of the latter option can be easily obtained from the above.

Recall that the payoff of a double-barrier step option is given by

$$
\mathrm{e}^{-\rho^{-} \int_{0}^{T} \mathbf{1}_{\left\{S_{t} \leq L\right\}} \mathrm{d} t-\rho^{+} \int_{0}^{T} \mathbf{1}_{\left\{S_{t} \geq U\right\}} \mathrm{d} t}\left(S_{T}-K\right)_{+},
$$

where $\rho^{-}$and $\rho^{+}$are the knock-out rates. Then, its price can be written as

$$
C^{\text {double }}(k, T):=\mathrm{e}^{-r T} \mathbb{E}\left[\mathrm{e}^{-\rho^{-} \int_{0}^{T} \mathbf{1}_{\left\{X_{t} \leq \ln \left(L / S_{0}\right)\right\}} \mathrm{d} t-\rho^{+} \int_{0}^{T} \mathbf{1}_{\left\{X_{t} \geq \ln \left(U / S_{0}\right)\right\}} \mathrm{d} t}\left(S_{0} \mathrm{e}^{X_{T}}-\mathrm{e}^{-k}\right)^{+}\right]
$$


where $k=-\ln (K)$. Again, we can easily compute its double Laplace transform:

$\int_{0}^{\infty} \int_{-\infty}^{\infty} \mathrm{e}^{-\alpha T-\beta k} C^{\text {double }}(k, T) \mathrm{d} k \mathrm{~d} T=\mathrm{e}^{-r T} \frac{S_{0}^{\beta+1} w\left(\ln \left(S_{0}\right) ; \ln \left(L / S_{0}\right), \ln \left(U / S_{0}\right) \alpha, \rho^{-}, \rho^{+}, \beta+1\right)}{\alpha \beta(\beta+1)}$

where an explicit expression for

$$
w\left(x ; h, H, \alpha, \rho^{-}, \rho^{+}, \gamma\right)=\int_{0}^{\infty} \alpha \mathrm{e}^{-\alpha T} \mathbb{E}_{x}\left[\mathrm{e}^{-\rho_{1} \int_{0}^{T} \mathbf{1}_{\left\{X_{t} \leq h\right\}} \mathrm{d} t-\rho_{2} \int_{0}^{T} \mathbf{1}_{\left\{X_{t} \geq H\right\}} \mathrm{d} t+\gamma X_{T}}\right] \mathrm{d} T
$$

is given by Theorem 3 ,

4.2. Quantile options. Recall from the Introduction that a fixed-strike $\alpha$-quantile call option admits the following payoff: for $0 \leq \alpha \leq 1$,

$$
\left(S_{0} \mathrm{e}^{\gamma q(\alpha, T)}-K\right)^{+}
$$

where

$$
q(\alpha, T)=\inf \left\{h: \int_{0}^{T} \mathbf{1}_{\left\{X_{t} \leq h\right\}} \mathrm{d} t>\alpha T\right\}
$$

For any $0 \leq v \leq T$, the price of this $\alpha$-quantile call option can be written as

$$
C^{\text {quantile }}(v, T)=\mathrm{e}^{-r T} \mathbb{E}\left[\left(S_{0} \mathrm{e}^{\lambda q(v / T, T)}-K\right)_{+}\right] .
$$

Then, the double Laplace transform of $C^{\text {quantile }}(v, T)$ is given by

$$
\begin{aligned}
\int_{0}^{\infty} \int_{0}^{\infty} \mathrm{e}^{-\alpha T-\rho v} C^{\text {quantile }}(v, T) \mathbf{1}_{\{v<T\}} \mathrm{d} T \mathrm{~d} v & \text { if } K \leq S_{0}, \\
= & \left\{\begin{array}{cc}
\sum_{i=1}^{m+1} \frac{\lambda K}{\rho} \frac{\omega_{i}}{\beta_{i, \alpha+\rho}-\lambda}\left(S_{0} / K\right)^{\left(\beta_{i, \alpha+\rho}+\lambda\right) / \lambda} & \\
\sum_{i=1}^{m+1} \frac{\lambda K}{\rho} \frac{\omega_{i}}{\beta_{i, \alpha+\rho}-\lambda}\left(S_{0} / K\right)^{\left(\beta_{i, \alpha+\rho}+\lambda\right) / \lambda} & \\
-\sum_{j=1}^{n+1} \frac{\lambda K}{\rho} \frac{\nu_{j}}{\gamma_{j, \alpha}+\lambda}\left(1-\left(S_{0} / K\right)^{\left(\gamma_{i, \alpha+\rho}+\lambda\right) / \lambda}\right)+\frac{\left(S_{0}-K\right)}{\alpha(\alpha+\rho)} & \text { if } K<S_{0},
\end{array}\right.
\end{aligned}
$$

where $\left\{\omega_{i}, i=1, \ldots, m+1\right\}$ and $\left\{\nu_{j}, j=1, \ldots, n+1\right\}$ are given by Theorem 2 ,

\section{AcKnowledgements}

Funding in support of this work was provided by an ENC grant from the Fonds de recherche du Québec - Nature et technologies (FRQNT). J.-F. Renaud also thanks the Institut de finance mathématique de Montréal (IFM2) for its financial support. 


\section{Proof of Theorem 2}

We have for $x<h$

$$
\begin{aligned}
w(x) & =\mathbb{E}_{x}\left[\mathrm{e}^{-\rho \int_{0}^{\mathbf{e} \alpha} \mathbf{1}_{\left\{h<X_{s}<H\right\}} \mathrm{d} s+\gamma X_{\mathbf{e}_{\alpha}}}\right] \\
& =\mathbb{E}_{x}\left[\mathrm{e}^{-\rho \int_{0}^{\mathbf{e}_{\alpha}} \mathbf{1}_{\left\{h<X_{s}<H\right\}} \mathrm{d} s+\gamma X_{\mathbf{e}_{\alpha}}}, \mathbf{e}_{\alpha}<\tau_{h}^{+}\right]+\mathbb{E}_{x}\left[\mathrm{e}^{-\rho \int_{0}^{\mathbf{e}_{\alpha}} \mathbf{1}_{\left\{h<X_{s} \leq H\right\}} \mathrm{d} s+\gamma X_{\mathbf{e}_{\alpha}}}, \tau_{h}^{+} \leq \mathbf{e}_{\alpha}\right] \\
& =\mathbb{E}_{x}\left[\mathrm{e}^{\gamma X_{\mathbf{e}_{\alpha}}}, \mathbf{e}_{\alpha}<\tau_{h}^{+}\right]+\mathbb{E}_{x}\left[\mathrm{e}^{-\rho \int_{0}^{\mathbf{e}_{\alpha}} \mathbf{1}_{\left\{h<X_{s} \leq H\right\}} \mathrm{d} s+\gamma X_{\mathbf{e}_{\alpha}}}, \tau_{h}^{+} \leq \mathbf{e}_{\alpha}\right] \\
(10) & =\mathbb{E}_{x}\left[\int_{0}^{\tau_{h}^{+}} \alpha \mathrm{e}^{-\alpha s+\gamma X_{s}} \mathrm{~d} s\right]+\mathbb{E}_{x}\left[\mathrm{e}^{-\rho \int_{0}^{\mathbf{e}_{\alpha}} \mathbf{1}_{\left\{h<X_{s} \leq H\right\}} \mathrm{d} s+\gamma X_{\mathbf{e}_{\alpha}}}, \tau_{h}^{+} \leq \mathbf{e}_{\alpha}\right] .
\end{aligned}
$$

Applying Itô's formula to the process $\left\{\mathrm{e}^{-\alpha t+\gamma X_{t}}, t \geq 0\right\}$, we obtain that the process

$$
\begin{aligned}
M_{t} & :=\mathrm{e}^{-\alpha\left(t \wedge \tau_{h}^{+}\right)+\gamma X_{t \wedge \tau_{h}^{+}}}-\mathrm{e}^{\gamma X_{0}}-\int_{0}^{t \wedge \tau_{h}^{+}} \mathrm{e}^{-\alpha s}\left(-\alpha \mathrm{e}^{\gamma X_{s}}+\mathcal{L} \mathrm{e}^{\gamma X_{s}}\right) \mathrm{d} s \\
& =\mathrm{e}^{-\alpha\left(t \wedge \tau_{h}^{+}\right)+\gamma X_{t \wedge \tau_{h}^{+}}}-\mathrm{e}^{\gamma x}-(G(\gamma)-\alpha) \int_{0}^{t \wedge \tau_{h}^{+}} \mathrm{e}^{-\alpha s+\gamma X_{s}} \mathrm{~d} s
\end{aligned}
$$

is a local martingale starting from $M_{0}=0$. Since $G(\gamma)<\alpha$, it follows from Fubini's theorem that

$$
\mathbb{E}\left[\int_{0}^{t} \mathrm{e}^{-\alpha s+\gamma X_{s}} \mathrm{~d} s\right]=\int_{0}^{t} \mathrm{e}^{-\alpha s} \mathbb{E}\left[\mathrm{e}^{\gamma X_{s}}\right] \mathrm{d} s=\int_{0}^{t} \mathrm{e}^{(-\alpha+G(\gamma)) s} \mathrm{~d} s=\frac{\mathrm{e}^{(-\alpha+G(\gamma)) t}-1}{(-\alpha+G(\gamma))}<\infty,
$$

for all $t \geq 0$. So, using Lebesgue's dominated convergence theorem, we have that $\left\{M_{t}, t \geq 0\right\}$ is actually a martingale. In particular,

$$
\mathbb{E}_{x}\left[\mathrm{e}^{-\alpha \tau_{h}^{+}+\gamma X_{\tau_{h}^{+}}}-\mathrm{e}^{\gamma x}\right]=(G(\gamma)-\alpha) \mathbb{E}_{x}\left[\int_{0}^{\tau_{h}^{+}} \mathrm{e}^{-\alpha s+\gamma X_{s}} \mathrm{~d} s\right]
$$

Plugging (11) into (10), we get, by the strong Markov property of $X$ and the lack-of-memory property of $\mathbf{e}_{\alpha}$, that

$$
w(x)=\frac{\alpha}{G(\gamma)-\alpha}\left(\mathbb{E}_{x}\left[\mathrm{e}^{-\alpha \tau_{h}^{+}+X_{\tau_{h}^{+}}}\right]-\mathrm{e}^{\gamma x}\right)+\mathbb{E}_{x}\left[\mathrm{e}^{-\alpha \tau_{h}^{+}} w\left(X_{\tau_{h}^{+}}\right)\right] .
$$

Similarly, for $x>H$,

$$
w(x)=\frac{\alpha}{G(\gamma)-\alpha}\left(\mathbb{E}_{x}\left[\mathrm{e}^{-\alpha \tau_{h}^{-}+\gamma X_{\tau_{h}^{-}}}\right]-\mathrm{e}^{\gamma x}\right)+\mathbb{E}_{x}\left[\mathrm{e}^{-\alpha \tau_{h}^{-}} w\left(X_{\tau_{h}^{-}}\right)\right],
$$

and, for $h \leq x \leq H$,

$$
w(x)=\frac{\alpha}{G(\gamma)-(\alpha+\rho)}\left(\mathbb{E}_{x}\left[\mathrm{e}^{-(\alpha+\rho) \tau+\gamma X_{\tau}}\right]-\mathrm{e}^{\gamma x}\right)+\mathbb{E}_{x}\left[\mathrm{e}^{-(\rho+\alpha) \tau} w\left(X_{\tau}\right)\right] .
$$

Define the following:

$$
w(x)= \begin{cases}w_{1}(x), & x \leq h \\ w_{2}(x), & h<x<H \\ w_{3}(x), & x \geq H\end{cases}
$$


Combining Theorem 1 with equations (12), (13) and (14), we get that $w(x)$ must be of the following form:

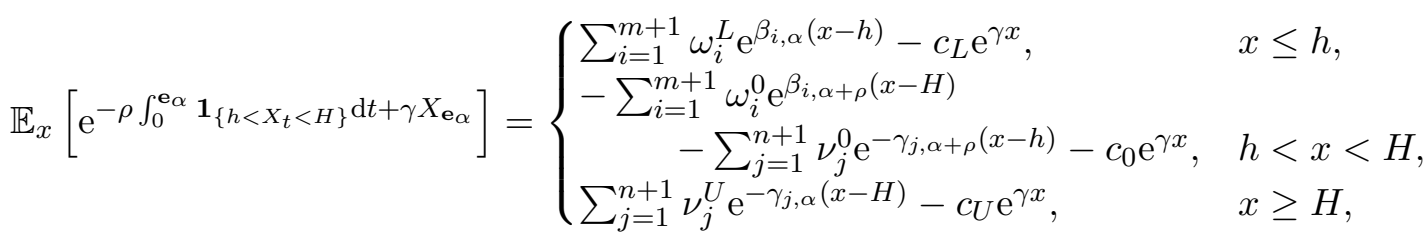

with $\omega_{i}^{L}, \omega_{i}^{0}, \nu_{j}^{0}$ and $\nu_{j}^{U}$ to be determined. Now, we need equations to determine these coefficients.

Using again equations (12), (13) and (14), we have that $w(x)$ must satisfy

$$
\left(\mathcal{L}-\alpha-\rho \mathbf{1}_{\{h<x<H\}}\right) w(x)=-\alpha \mathrm{e}^{\gamma x}, \quad x \in \mathbb{R} \backslash\{h, H\} .
$$

Then, equation (16) can be rewritten as three separate equations in the regions $(-\infty, h),(h, H)$ and $(H,+\infty)$.

For $x<h$,

$$
\begin{aligned}
&-\alpha \mathrm{e}^{\gamma x}= \frac{\sigma^{2}}{2} w_{1}^{\prime \prime}(x)+\mu w_{1}^{\prime}(x)-(\lambda+\alpha) w_{1}(x) \\
&+\lambda\left\{\int_{-\infty}^{0} w_{1}(x+y) \sum_{j=1}^{n} q_{j} \theta_{j} \mathrm{e}^{\theta_{j} y} \mathrm{~d} y+\int_{0}^{h-x} w_{1}(x+y) \sum_{j=1}^{m} p_{j} \eta_{j} \mathrm{e}^{-\eta_{j} y} \mathrm{~d} y\right. \\
&\left.\quad+\int_{h-x}^{H-x} w_{2}(x+y) \sum_{j=1}^{m} p_{j} \eta_{j} \mathrm{e}^{-\eta_{j} y} \mathrm{~d} y+\int_{H-x}^{+\infty} w_{3}(x+y) \sum_{j=1}^{m} p_{j} \eta_{j} \mathrm{e}^{-\eta_{j} y} \mathrm{~d} y\right\} .
\end{aligned}
$$

For $h<x<H$,

$$
\begin{aligned}
&-\alpha \mathrm{e}^{\gamma x}= \frac{\sigma^{2}}{2} w_{2}^{\prime \prime}(x)+\mu w_{2}^{\prime}(x)-(\lambda+\rho+\alpha) w_{2}(x) \\
&+\lambda\left\{\int_{-\infty}^{h-x} w_{1}(x+y) \sum_{j=1}^{n} q_{j} \theta_{j} \mathrm{e}^{\theta_{j} y} \mathrm{~d} y+\int_{h-x}^{0} w_{2}(x+y) \sum_{j=1}^{n} q_{i} \theta_{i} \mathrm{e}^{\theta_{i} y} \mathrm{~d} y\right. \\
&\left.\quad+\int_{0}^{H-x} w_{2}(x+y) \sum_{j=1}^{m} p_{j} \eta_{j} \mathrm{e}^{-\eta_{j} y} \mathrm{~d} y+\int_{H-x}^{+\infty} w_{3}(x+y) \sum_{j=1}^{m} p_{j} \eta_{j} \mathrm{e}^{-\eta_{j} y} \mathrm{~d} y\right\} .
\end{aligned}
$$

And finally, for $x>H$,

$$
\begin{aligned}
&-\alpha \mathrm{e}^{\gamma x}= \frac{\sigma^{2}}{2} w_{3}^{\prime \prime}(x)+\mu w_{3}^{\prime}(x)-(\lambda+\alpha) w_{3}(x) \\
&+\lambda\left\{\int_{-\infty}^{h-x} w_{1}(x+y) \sum_{j=1}^{n} q_{j} \theta_{j} \mathrm{e}^{\theta_{j} y} \mathrm{~d} y+\int_{h-x}^{H-x} w_{2}(x+y) \sum_{j=1}^{n} q_{i} \theta_{i} \mathrm{e}^{\theta_{i} y} \mathrm{~d} y\right. \\
&\left.\quad+\int_{H-x}^{0} w_{3}(x+y) \sum_{j=1}^{n} q_{i} \theta_{i} \mathrm{e}^{\theta_{i} y} \mathrm{~d} y+\int_{0}^{+\infty} w_{3}(x+y) \sum_{j=1}^{m} p_{j} \eta_{j} \mathrm{e}^{-\eta_{j} y} \mathrm{~d} y\right\} .
\end{aligned}
$$


Substituting the expression obtained in (15) into (17), (18) and (19), we get, for $x<h$,

$$
\begin{gathered}
0=\sum_{j=1}^{m} p_{j} \eta_{j} \mathrm{e}^{\eta_{j}(x-h)}\left\{\sum_{i=1}^{m+1}\left(\frac{\omega_{i}^{L}}{\eta_{j}-\beta_{i, \alpha}}+\frac{\omega_{i}^{0} \mathrm{e}^{\beta_{i, \alpha+\rho}(h-H)}}{\eta_{j}-\beta_{i, \alpha+\rho}}\right)+\sum_{i=1}^{n+1}\left(\frac{\nu_{i}^{0}}{\eta_{j}+\gamma_{i, \alpha+\rho}}\right)-\left(c_{L}-c_{0}\right) \frac{\mathrm{e}^{\gamma h}}{\eta_{j}-\gamma}\right\} \\
+\sum_{j=1}^{m} p_{j} \eta_{j} \mathrm{e}^{\eta_{j}(x-H)}\left\{\sum_{i=1}^{m+1}\left(\frac{\omega_{i}^{0}}{\eta_{j}-\beta_{i, \alpha+\rho}}\right)+\sum_{i=1}^{n+1}\left(\frac{\nu_{i}^{0} \mathrm{e}^{-\gamma_{i, \alpha+\rho}(h-H)}}{\eta_{j}+\gamma_{i, \alpha+\rho}}+\frac{\nu_{i}^{U}}{\eta_{j}+\gamma_{i, \alpha}}\right)-\left(c_{L}-c_{0}\right) \frac{\mathrm{e}^{\gamma H}}{\eta_{j}-\gamma}\right\} .
\end{gathered}
$$

and, for $x>H$,

$$
\begin{array}{r}
0=\sum_{j=1}^{n} q_{j} \theta_{j} \mathrm{e}^{\theta_{j}(h-x)}\left\{\sum_{i=1}^{m+1}\left(\frac{\omega_{i}^{L}}{\theta_{j}+\beta_{i, \alpha}}+\frac{\omega_{i}^{0} \mathrm{e}^{\beta_{i, \alpha+\rho}(h-H)}}{\theta_{j}+\beta_{i, \alpha+\rho}}\right)+\sum_{i=1}^{n+1}\left(\frac{\nu_{i}^{0}}{\theta_{j}-\gamma_{i, \alpha+\rho}}\right)-\left(c_{L}-c_{0}\right) \frac{\mathrm{e}^{\gamma h}}{\theta_{j}+\gamma}\right\} \\
+\sum_{j=1}^{n} q_{j} \theta_{j} \mathrm{e}^{\theta_{j}(H-x)}\left\{\sum_{i=1}^{m+1}\left(\frac{\omega_{i}^{0}}{\theta_{j}+\beta_{i, \alpha+\rho}}\right)+\sum_{i=1}^{n+1}\left(\frac{-\nu_{i}^{0} \mathrm{e}^{-\gamma_{i, \alpha+\rho}(h-H)}}{\theta_{j}-\gamma_{i, \alpha+\rho}}+\frac{\nu_{i}^{U}}{\theta_{j}-\gamma_{i, \alpha}}\right)-\left(c_{L}-c_{0}\right) \frac{\mathrm{e}^{\gamma H}}{\theta_{j}+\gamma}\right\} .
\end{array}
$$

Therefore, the vector $Q$ or, in other words, the coefficients $\left\{\omega_{i}^{L}, i=1, \ldots, m+1\right\},\left\{\omega_{i}^{0}, i=\right.$ $1, \ldots, m+1\},\left\{\nu_{i}^{0}, i=1, \ldots, n+1\right\}$ and $\left\{\nu_{i}^{U}, i=1, \ldots, n+1\right\}$ satisfy the following: for each $j=1, \ldots, m$,

$$
\begin{aligned}
& 0=\sum_{i=1}^{m+1}\left(\frac{\omega_{i}^{L}}{\eta_{j}-\beta_{i, \alpha}}+\frac{\omega_{i}^{0} \mathrm{e}^{\beta_{i, \alpha+\rho}(h-H)}}{\eta_{j}-\beta_{i, \alpha+\rho}}\right)+\sum_{i=1}^{n+1}\left(\frac{\nu_{i}^{0}}{\eta_{j}+\gamma_{i, \alpha+\rho}}\right)-\left(c_{L}-c_{0}\right) \frac{\mathrm{e}^{\gamma h}}{\eta_{j}-\gamma}, \\
& 0=\sum_{i=1}^{m+1}\left(\frac{\omega_{i}^{0}}{\eta_{j}-\beta_{i, \alpha+\rho}}\right)+\sum_{i=1}^{n+1}\left(\frac{-\nu_{i}^{0} \mathrm{e}^{-\gamma_{i, \alpha+\rho}(h-H)}}{\eta_{j}+\gamma_{i, \alpha+\rho}}+\frac{\nu_{i}^{U}}{\eta_{j}+\gamma_{i, \alpha}}\right)-\left(c_{L}-c_{0}\right) \frac{\mathrm{e}^{\gamma H}}{\eta_{j}-\gamma},
\end{aligned}
$$

and, for each $j=1, \ldots, n$,

$$
\begin{aligned}
& 0=\sum_{i=1}^{m+1}\left(\frac{\omega_{i}^{L}}{\theta_{j}+\beta_{i, \alpha}}+\frac{\omega_{i}^{0} \mathrm{e}^{\beta_{i, \alpha+\rho}(h-H)}}{\theta_{j}+\beta_{i, \alpha+\rho}}\right)+\sum_{i=1}^{n+1}\left(\frac{\nu_{i}^{0}}{\theta_{j}-\gamma_{i, \alpha+\rho}}\right)-\left(c_{L}-c_{0}\right) \frac{\mathrm{e}^{\gamma h}}{\theta_{j}+\gamma}, \\
& 0=\sum_{i=1}^{m+1}\left(\frac{\omega_{i}^{0}}{\theta_{j}+\beta_{i, \alpha+\rho}}\right)+\sum_{i=1}^{n+1}\left(\frac{-\nu_{i}^{0} \mathrm{e}^{-\gamma_{i, \alpha+\rho}(h-H)}}{\theta_{j}-\gamma_{i, \alpha+\rho}}+\frac{\nu_{i}^{U}}{\theta_{j}-\gamma_{i, \alpha}}\right)-\left(c_{L}-c_{0}\right) \frac{\mathrm{e}^{\gamma H}}{\theta_{j}+\gamma} .
\end{aligned}
$$

In addition, we also have the following four equations:

$$
\begin{aligned}
& \sum_{i=1}^{m+1} \omega_{i}^{L}-c_{L} \mathrm{e}^{\gamma h}=\sum_{i=1}^{m+1}-\omega_{i}^{0} \mathrm{e}^{\beta_{i, \rho+\alpha}(h-H)}-\sum_{i=1}^{n+1} \nu_{i}^{0}-c_{0} \mathrm{e}^{\gamma h} \\
& \sum_{i=1}^{n+1} \nu_{i}^{U}-c_{U} \mathrm{e}^{\gamma H}=\sum_{i=1}^{m+1}-\omega_{i}^{0}-\sum_{i=1}^{n+1} \nu_{i}^{0} \mathrm{e}^{-\gamma_{i, \rho+\alpha}(H-h)}-c_{0} \mathrm{e}^{\gamma H}, \\
& \sum_{i=1}^{m+1} \omega_{i}^{L} \beta_{i, \alpha}-c_{L} \gamma \mathrm{e}^{\gamma h}=\sum_{i=1}^{m+1}-\omega_{i}^{0} \beta_{i, \alpha+\rho} \mathrm{e}^{\beta_{i, \rho+\alpha}(h-H)}+\sum_{i=1}^{n+1} \nu_{i}^{0} \gamma_{i, \alpha+\rho}-c_{0} \gamma \mathrm{e}^{\gamma h}, \\
& \sum_{i=1}^{n+1}-\nu_{i}^{U} \gamma_{i, \alpha}-c_{U} \gamma \mathrm{e}^{\gamma H}=\sum_{i=1}^{m+1}-\omega_{i}^{0} \beta_{i, \alpha+\rho}+\sum_{i=1}^{n+1} \nu_{i}^{0} \gamma_{i, \alpha+\rho} \mathrm{e}^{-\gamma_{i, \rho+\alpha}(H-h)}-c_{0} \gamma \mathrm{e}^{\gamma H}
\end{aligned}
$$


Indeed, equations (20) and (21) are immediate from the fact that $w(x)$ is continuous at $x=h$ and $x=H$.

For the proofs of equations (22) and (23), note that we have, for $h<x<H$,

$$
\begin{aligned}
-\alpha \mathrm{e}^{\gamma x}= & \frac{\sigma^{2}}{2} w_{2}^{\prime \prime}(x)+\mu w_{2}^{\prime}(x)-(\lambda+\rho+\alpha) w_{2}(x) \\
& +\lambda\left\{\int_{-\infty}^{h-x} w_{1}(x+y) \sum_{j=1}^{n} q_{j} \theta_{j} \mathrm{e}^{\theta_{j} y} \mathrm{~d} y+\int_{h-x}^{0} w_{2}(x+y) \sum_{j=1}^{n} q_{i} \theta_{i} \mathrm{e}^{\theta_{i} y} \mathrm{~d} y\right. \\
& \left.\quad+\int_{0}^{H-x} w_{2}(x+y) \sum_{j=1}^{m} p_{j} \eta_{j} \mathrm{e}^{-\eta_{j} y} \mathrm{~d} y+\int_{H-x}^{+\infty} w_{3}(x+y) \sum_{j=1}^{m} p_{j} \eta_{j} \mathrm{e}^{-\eta_{j} y} \mathrm{~d} y\right\} .
\end{aligned}
$$

Substituting expressions for $w_{1}(x), w_{2}(x)$ and $w_{3}(x)$ yields, for $h<x<H$,

$$
\begin{gathered}
-\alpha \mathrm{e}^{\gamma x}=-\sum_{i=1}^{m+1} \mathrm{e}^{\beta_{i, \alpha+\rho}(x-H)} \omega_{i}^{0}\left(\frac{\sigma^{2}}{2} \beta_{i, \alpha+\rho}^{2}-\mu \beta_{i, \alpha+\rho}-(\lambda+\alpha+\rho)\right) \\
-\sum_{i=1}^{n+1} \mathrm{e}^{\gamma_{i, \alpha+\rho}(x-h)} \nu_{j}^{0}\left(\frac{\sigma^{2}}{2} \gamma_{i, \alpha+\rho}^{2}-\mu \gamma_{i, \alpha+\rho}-(\lambda+\alpha+\rho)\right) \\
+\lambda\left\{\int_{-\infty}^{h-x} \sum_{i=1}^{m+1} \mathrm{e}^{\beta_{i, \alpha}(x+y-h)} \omega_{i}^{L} \sum_{j=1}^{n} q_{j} \theta_{j} \mathrm{e}^{\theta_{j} y} \mathrm{~d} y-\int_{h-x}^{0} \sum_{i=1}^{m+1} \mathrm{e}^{\beta_{i, \alpha+\rho}(x+y-H)} \omega_{i}^{0} \sum_{j=1}^{n} q_{j} \theta_{j} \mathrm{e}^{\theta_{j} y} \mathrm{~d} y\right. \\
-\int_{h-x}^{0} \sum_{i=1}^{n+1} \mathrm{e}^{\gamma_{i, \alpha+\rho}(x+y-h)} \nu_{i}^{0} \sum_{j=1}^{n} q_{j} \theta_{j} \mathrm{e}^{\theta_{j} y} \mathrm{~d} y-\int_{0}^{H-x} \sum_{i=1}^{m+1} \omega_{i}^{0} \mathrm{e}^{\beta_{i, \alpha+\rho}(x+y-H)} \sum_{j=1}^{m} p_{j} \eta_{j} \mathrm{e}^{-\eta_{j} y} \mathrm{~d} y \\
-\int_{0}^{H-x} \sum_{j=1}^{n+1} \nu_{j}^{0} \mathrm{e}^{-\gamma_{j, \alpha+\rho}(x-h)} \sum_{j=1}^{m} p_{j} \eta_{j} \mathrm{e}^{-y\left(\eta_{j}+\gamma_{j, \alpha}\right)} \mathrm{d} y \\
\left.+\int_{H-x}^{+\infty} \sum_{j=1}^{n+1} \nu_{j}^{U} \mathrm{e}^{-\gamma_{j, \alpha}(x+y-H)} \sum_{j=1}^{m} p_{j} \eta_{j} \mathrm{e}^{-\eta_{j} y} \mathrm{~d} y\right\} \\
-\lambda\left\{\begin{array}{c}
c_{L} \int_{-\infty}^{h-x} \mathrm{e}^{\gamma(x+y)} \sum_{j=1}^{n} q_{j} \theta_{j} \mathrm{e}^{\theta_{j} y} \mathrm{~d} y-c_{0} \int_{-\infty}^{h-x} \mathrm{e}^{\gamma(x+y)} \sum_{j=1}^{n} q_{j} \theta_{j} \mathrm{e}^{\theta_{j} y} \mathrm{~d} y \\
\left.c^{\gamma(x+y)} \sum_{j=1}^{m} p_{j} \eta_{j} \mathrm{e}^{-\eta_{j} y} \mathrm{~d} y+c_{U} \int_{H-x}^{+\infty} \mathrm{e}^{\gamma(x+y)} \sum_{j=1}^{m} p_{j} \eta_{j} \mathrm{e}^{-\eta_{j} y} \mathrm{~d} y\right\}
\end{array}\right.
\end{gathered}
$$

Since

$$
-c_{0}(\mathcal{L}-\alpha-\rho) \mathrm{e}^{\gamma x}=-\alpha \mathrm{e}^{\gamma x},
$$

and

$$
G\left(\beta_{i, \alpha+\rho}\right)-\alpha-\rho=G\left(\gamma_{j, \alpha+\rho}\right)-\alpha-\rho=0,
$$


then computing the second derivative of Equation (24) with respect to $x$ yields (23) and (23). The proof of Theorem 2 is complete.

\section{REFERENCES}

[1] N. Cai, On first passage times of a hyper-exponential jump diffusion process, Oper. Res. Lett. 37 (2009), no. 2, 127-134.

[2] N. Cai, N. Chen, and X. Wan, Occupation times of jump-diffusion processes with double exponential jumps and the pricing of options, Math. Oper. Res. 35 (2010), no. 2, 412-437.

[3] N Cai and S. G. Kou, Option pricing under a mixed-exponential jump diffusion model, Management Science 57 (2011), no. 11, 2067-2081.

[4] P. Carr and D. B. Madan, Option valuation using the fast Fourier transform, J. Comput. Finance 2 (1999), $61-73$.

[5] Y.-T. Chen, Y.-C. Sheu, and M.-C. Chang, A note on first passage functionals for hyper-exponential jumpdiffusion processes, Electron. Commun. Probab. 18 (2013), 1-8.

[6] D. Davydov and V. Linetsky, Structuring, pricing and hedging double-barrier step options, J. Comput. Finance 5 (2002), no. 2, 55-86.

[7] G. Fusai, Corridor options and arc-sine law, Ann. Appl. Probab. 10 (2000), no. 2, 634-663.

[8] H. Geman and M. Yor, Pricing and hedging double-barrier options: A probabilistic approach, Math. Finance 6 (1996), no. 4, 365-378.

[9] J.-N. Hugonnier, The Feynman-Kac formula and pricing occupation time derivatives, Int. J. Theor. Appl. Finance 2 (1999), no. 2, 153-178.

[10] S. Kou, G. Petrella, and H. Wang, Pricing path-dependent options with jump risk via Laplace transforms, The Kyoto Economic Review 74 (2005), no. 1, 1-23.

[11] S. G. Kou, A jump-diffusion model for option pricing, Management Science 48 (2002), no. 8, $1086-1101$.

[12] K. S. Leung and Y. K. Kwok, Distribution of occupation times for constant elasticity of variance diffusion and the pricing of $\alpha$-quantile options, Quant. Finance 7 (2007), no. 1, 87-94.

[13] V. Linetsky, Step options, Math. Finance 9 (1999), no. 1, 55-96.

[14] R. C. Merton, Option pricing when underlying stock returns are discontinuous, Journal of Financial Economics 3 (1976), 125-144.

[15] R. Miura, A note on lookback options based on order statistics, Hitotsubashi Journal of Commerce and Management 27 (1992), 15-28.

[16] A. Pechtl, Distributions of occupation times of Brownian motion with drift, J. Appl. Math. Decis. Sci. 3 (1999), no. 1, 41-62.

Quantact \& Département de mathématiques, Université du Québec à Montréal (UQAM), 201 aV. Président-Kennedy, Montréal (Québec) H2X 3Y7, Canada

E-mail address: ait_aoudia.djilali@courrier.uqam.ca

Département de mathématiques, Université du Québec à Montréal (UQAM), 201 av. PrésidentKennedy, Montréal (Québec) H2X 3Y7, Canada

E-mail address: renaud.jf@uqam.ca 